\title{
The Relationship between Place Attachment and Social Well-Being in Older Adults
}

\author{
Pouya Farokhnezhad Afshar ${ }^{1}$; Mahshid Foroughan ${ }^{2, *} ;$ AbouAli Vedadhir ${ }^{3}$; Mahmoud Ghazi \\ Tabatabaei ${ }^{3}$
}

${ }^{1} \mathrm{PhD}$ Candidate in Gerontology, Iranian Research Center On Aging, University of Social Welfare and Rehabilitation Science, Tehran, Iran

${ }^{2}$ Iranian Research Center of Aging, University of Social Welfare and Rehabilitation Science, Tehran, Iran

${ }^{3}$ Faculty of Social Science, Department of Anthropology, University of Tehran, Tehran, Iran

*Corresponding author: Mahshid Foroughan, Iranian Research Center of Aging, University of Social Welfare and Rehabilitation Science, Kodakyar Ave., Daneshjo Blvd., Evin, Tehran, Iran. Tel: +98-21222180004 Email: m_foroughan@yahoo.com

Received: 22 Sep 2016

Accepted: 21 Nov 2016

Ppub: 23 Dec 2016

\begin{abstract}
Background: Health has been defined as a state of complete physical, psychological, and social well-being and place attachment has an effect on self-rated health of older adults. Neighborhood is a part of the social life of each person and his or her feeling to the neighborhood is important in well-being. There are few studies on the relationship between place attachment and social wellbeing.

Methods: This study was a descriptive and analytical study conducted on 550 older adults in a Tehran city. The Social Well-being Scale and the Place Attachment Scale were used to collect data. Data analysis was performed using Pearson correlation and multiple regression analysis by IBM SPSS statistics V.22 software.

Results: Findings showed that place attachment, explained $(\beta=.78, \mathrm{P}<.001)$ of the variance in social well-being. The finding of this study revealed that the place attachment has a strong effect on social well-being of older adults.

Conclusions: Any change in the place of attachment leads to changes in the social well-being and finally on health in the older adults. So, the improvement of the living place has a major role on health and well-being.
\end{abstract}

Keywords: Health; Social Determinants of Health; Older Adults 